\title{
Prevalence of Helicobacter pylori infection in patients with chronic hepatitis $C$
}

\author{
Abdelfattah M. Attallah', Mohamed S. Albannan ${ }^{1 *}$, Mohamed F. Ghaly², Sally E. Sallam³, \\ Mahmoud M. Amer ${ }^{3}$ and Attia A. Attia ${ }^{3}$
}

\begin{abstract}
Background: Association between Helicobacter pylori (H. pylori) and chronic hepatitis C (CHC) still remains controversial. This work is concerned with assessing the potential role of $H$. pylori in the progression of hepatitis $\mathrm{C}$ virus (HCV)related chronic liver disease.

Results: A total of 449 individuals constituted this study (200 individuals were used to validate the assay while 249 individuals were used to assess the correlation between H. pylori infection and CHC). H. pylori antigen was quantified in serum samples using ELISA. As a consequence, our findings showed that H. pylori positivity was increased significantly $(P=0.021)$ with liver fibrosis progression as it was found in $44.45 \%$ of fibrotic patients and $71.88 \%$ of cirrhotic patients. We demonstrated that patients with $\mathrm{F} 4$ were accompanied by a significant $(P<0.05)$ increase in the concentration of H. pylori antigen displaying 16.52-fold and 1.34-fold increase in its level over F0 and F1-F3, respectively. Patients co-infected with H. pylori and HCV are 3.19 times (219\%) more likely to experience cirrhosis than those who are mono-infected with HCV. This suggests that the risk for developing F4 was found to increase upon H. pylori coinfection when compared to $\mathrm{CHC}$ mono-infected patients.
\end{abstract}

Conclusion: The elevated levels of H. pylori-antigen in HCV/H. pylori co-infection suggest increased susceptibility of co-infected patients for promoting hepatic fibrosis progression.

Keywords: Helicobacter pylori, Co-infection, Liver, Fibrosis, Cirrhosis

\section{Background}

The discovery of Helicobacter pylori (H. pylori) has without doubt been one of the most remarkable achievements in medical research in the past three decades. H. pylori infects $50 \%$ of the world population. Its prevalence varies widely in different parts of the world with higher rates in developing countries. In most instances, it is acquired during childhood, and is often associated with low socioeconomic class $[1,2]$. It produces chronic gastritis by provoking local inflammatory response in the epithelium through release of a range of cytokine [3]. The World Health Organization classifies the bacterium as a class I

\footnotetext{
*Correspondence: mohamedalbannan@yahoo.com

${ }^{1}$ Biotechnology Research Center, 23 July St., Industrial Zone, New

Damietta 34517, Egypt

Full list of author information is available at the end of the article
}

carcinogen and a close association between infection and $70 \%$ of gastric cancer cases worldwide has been reported $[1,2]$. Additionally, liver is likely a probable organ to be affected by $H$. pylori activity [4-6]. In this case, clinicians would need a rapid and reproducible, noninvasive assay for the screening of patients for the presence of this pathogen and if a patient indeed tested positive for $H$. pylori for monitoring the success of eradication therapy [7]. Unfortunately, H. pylori is fastidious and difficult to cultivate [8]. Therefore, detecting serum $H$. pylori antigen offers an alternative noninvasive diagnostic test. In this respect, we previously developed a sensitive and specific noninvasive immunoassay based on the detection of $H$. pylori circulating antigen in sera from $H$. pylori-infected individuals with high degree of accuracy [9]. On the other hand, increasing evidence suggests that $H$. pylori 
may be a risk factor for developing cirrhosis in patients with chronic hepatitis $\mathrm{C}(\mathrm{CHC})[10,11]$.

Herein, we are concerned with achieving two objectives. The first one is concerned with identification and quantitation of $\mathrm{H}$. pylori antigen in $\mathrm{CHC}$ patients and validate whether the predictive criteria identified in the previous study were able to reproduce their predictive ability in a subsequent different, but related, group of patients. The second one is dedicated to assessing the potential role of $\mathrm{H}$. pylori infection in the progression of hepatitis $\mathrm{C}$ virus ( $\mathrm{HCV}$ )-related chronic liver disease.

\section{Methods}

\section{Samples}

A total of 449 consecutive Egyptian individuals composed of three different groups constituted the present study. Informed consents were obtained from all participants and they were fully informed concerning the diagnostic procedures involved and disease nature. The study protocol was approved by the Ethics Committee of the Mansoura University hospitals, Mansoura University, Egypt. The consent to participate was obtained verbally and approved by Ethics Committee. The first group included serum samples of $100 \mathrm{H}$. pylori-infected individuals collected from the Gastro-Enterology and Surgery Center, Mansoura University, Mansoura, Egypt. They were diagnosed based on culture as a gold standard.

The second group included serum samples of another 249 chronic hepatitis $C$ patients [95 with F0, 90 with F1-F3, and 64 with F4] collected from the Tropical Medicine Department, Mansoura University hospitals, Mansoura, Egypt. These patients were tested positive for the presence of HCV-ribonucleic acid (RNA) using quantitative polymerase chain reaction assay (COBAS Ampliprep/COBAS TaqMan, Roche Diagnostics, Pleasanton, USA). Histopathological classification for liver fibrosis and cirrhosis was performed according to the METAVIR score [12]. Liver fibrosis was defined as a METAVIR score of $\leq 3$ (F1-F3) whereas cirrhosis was defined as a METAVIR score of 4 (F4). The third group included serum samples of another 100 healthy volunteers used as a control group. Overall, the first and the third groups were used to validate the assay while the second group was dedicated to assessing the correlation between $H$. pylori infection and chronic hepatitis $\mathrm{C}$.

\section{Laboratory tests}

All serum samples constituted this study were subjected to laboratory investigations including liver function tests [alanine aminotransferase (ALT), aspartate aminotransferase (AST), total bilirubin, albumin, and alkaline phosphatase (ALP)] measured on an automated biochemistry analyzer (A15, Biosystem, Spain). Alpha fetoprotein (AFP) level was performed by chemiluminescence, with Immulite AFP (1000) kit (Diagnostic Products Corporation; Los Angeles, CA, USA).

\section{Detection of 58-kDa H. pylori antigen using ELISA}

First of all, the target $58-\mathrm{kDa} H$. pylori antigen was previously identified at $58-\mathrm{kDa}$ molecular weight based on sodium dodecyl sulfate-polyacrylamide gel electrophoresis followed by Western blot as published by Attallah et al. [9]. In this work, the quantification of $58-\mathrm{kDa} H$. pylori antigen was performed based on ELISA technique using its mono-specific antibodies (ABC Diagnostics, New Damietta, Egypt) as according to Attallah et al. [9]. Color intensity was proportional to the amount of bound conjugate and therefore is a function of the concentration of $H$. pylori antigen present in the serum sample.

\section{Statistical analysis}

All statistical calculations were done by the SPSS software v.15.0 (SPSS Inc., Chicago, IL) and GraphPad Prism package v.5.0 (GraphPad Software, San Diego, CA). Continuous variables were expressed as mean \pm standard error of mean. One-way analysis of variance (ANOVA) and Tukey's post hoc test were used to compare continuous variables while chi-square test was used to compare proportions. All tests were two-tailed and statistical significance assessed at the 0.05 level. Area under the curve (AUC) was used to assess the diagnostic value of $58-\mathrm{kDa}$ H. pylori antigen to distinguish between patients infected with $H$. pylori and non-infected individuals. An AUC equal to 1.0 is characteristic of an ideal test, whereas 0.5 indicates a test without diagnostic value. The nearer a curve shifts to the top left-hand corner of the graph, the more useful the marker is for the diagnosis. Results obtained by the $H$. pylori culture test as a gold standard; common indicators of 58-kDa H. pylori antigen accuracy (sensitivity, specificity, efficiency, and odds ratio) were derived from such a $2 \times 2$ contingency table. Odds ratio (with 95\% confidence intervals) was calculated to estimate the risk of a target disorder from subjects without it.

\section{Results}

As formerly mentioned, the first objective of this work is to validate whether the predictive criteria identified in the previous study [9] were able to reproduce their predictive ability in a subsequent different, but related, group of patients based on ELISA analysis. In order to estimate the diagnostic accuracy of $58-\mathrm{kDa} H$. pylori antigen, ROC curve was used as depicted in Fig. 1. As a result, the 58-kDa $H$. pylori antigen enabled the correct identification of $H$. pylori patients showing an AUC of 0.946 with 90\% sensitivity, $90 \%$ specificity, and $90 \%$ efficiency. 


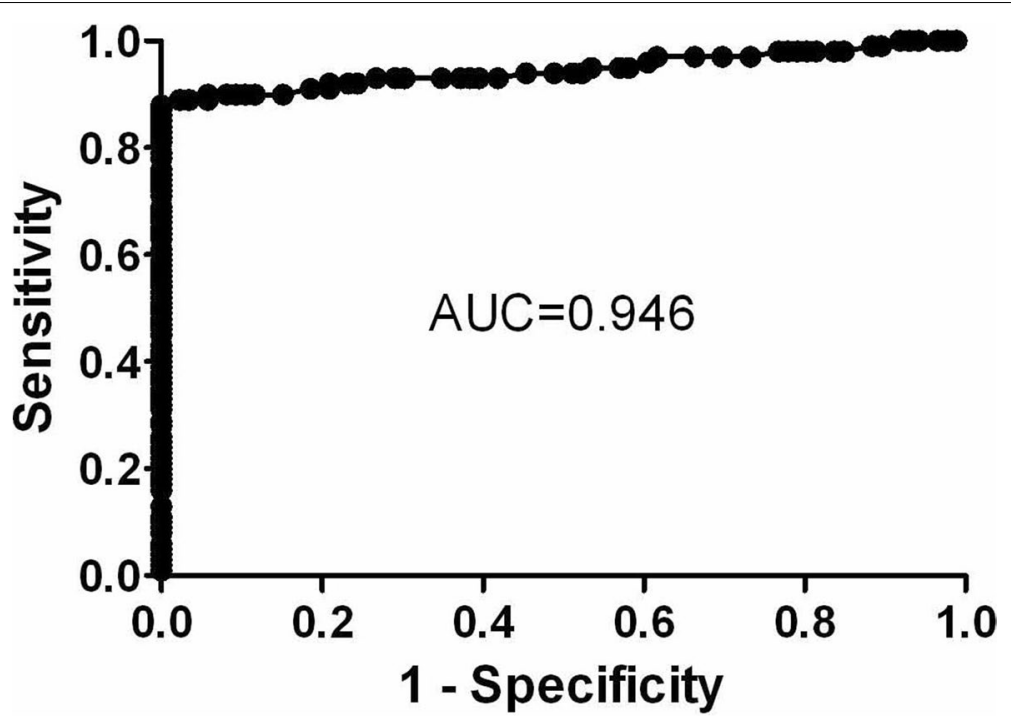

Fig. 1 Diagnostic accuracy of 58-kDa Helicobacter Pylori (H. pylori) antigen for the diagnosis of H. pylori infection

The levels of $H$. pylori antigen were then quantified in patients' sera and the results are depicted in Fig. 2a. As a consequence, our findings demonstrated that patients who had hepatic cirrhosis were accompanied by a significant $(P<0.05)$ increase in the concentration of $H$. pylori antigen when compared to those with F0 and F1-F3. Interestingly, patients with F1-F3 displayed 12.3fold increase in $H$. pylori antigen level over patients with F0 (Fig. 2b). On the other hand, cirrhotic patients displayed a 16.52-fold and 1.34-fold increase in H. pylori antigen level over F0 and F1-F3, respectively, as shown in Fig. 2c, d.

Laboratory characteristics of $\mathrm{CHC}$ patients with and without $H$. pylori infection are summarized in Table 1. Overall, there were $95 / 249$ (38.15\%) with no fibrosis (F0), 90/249 (36.15\%) with liver fibrosis (F1-F3), and 64/249 (25.70\%) with liver cirrhosis (F4) as seen in Table 2. Next, the characteristics of patients with no fibrosis (F0), liver fibrosis (F1-F3), and liver cirrhosis (F4) are summarized in Table 2. In this work, our patients were predominantly male $(79.19 \%)$, with a mean $( \pm S D)$ age of $39.63( \pm 11.61)$ years at the time of liver biopsy. Patients with no fibrosis (F0) were young as compared to those who developed liver fibrosis (F1-F3) or liver cirrhosis (F4) with an extremely significant difference $(P<0.0001)$ based on the analysis of one-way ANOVA followed by Tukey's post hoc test as presented in Table 2. As anticipated, patients who developed liver cirrhosis (F4) were associated with higher levels of alanine aminotransferase (ALT), aspartate aminotransferase (AST), and alpha fetoprotein (AFP) than those who have liver fibrosis (F1-F3) or those with no fibrosis $(\mathrm{F} 0)$ with a significant difference $(P<0.05)$.
On the other hand, the mean value of albumin and platelet count decreased with the progression of liver fibrosis being lower in patients who developed liver cirrhosis (F4) than those with no liver fibrosis (F0) and liver fibrosis (F1-F3).

Furthermore, the prevalence of $58-\mathrm{kDa} H$. pylori antigen in this category of patients was found to increase with the progression of liver disease as it was $44.45 \%$ in liver fibrosis and $71.88 \%$ in liver cirrhosis as shown in Fig. 2e. Our findings showed that $H$. pylori antigen significantly correlated with liver fibrosis stages producing a Spearman's rank correlation coefficient $(r)$ of $0.501(P<$ 0.0001) as depicted in Fig. $2 \mathrm{f}$.

Levels of different biochemical markers were determined in CHC patients with and without $H$. pylori as shown in Table 3 . The results demonstrated that each METAVIR fibrosis group was accompanied by an increase in their levels in case of co-infection compared to patients at the same stage in mono-infection. On the other hand, each fibrosis group was associated with a drop in the levels of both albumin and platelet count in case of co-infection compared to HCV mono-infection but the difference was statistically not significant.

Next, odds ratio was used as a measure of association between $58-\mathrm{kDa} H$. pylori antigen and different groups of diseases and the results are shown in Table 4. The risk for developing liver fibrosis and cirrhosis was found to increase upon $H$. pylori co-infection when compared to $\mathrm{CHC}$ mono-infected patients. Our findings showed that patients co-infected with $\mathrm{H}$. pylori and $\mathrm{HCV}$ are 3.19 times $(219 \%)$ more likely to experience cirrhosis than those who mono-infected with HCV. This may give a clue 

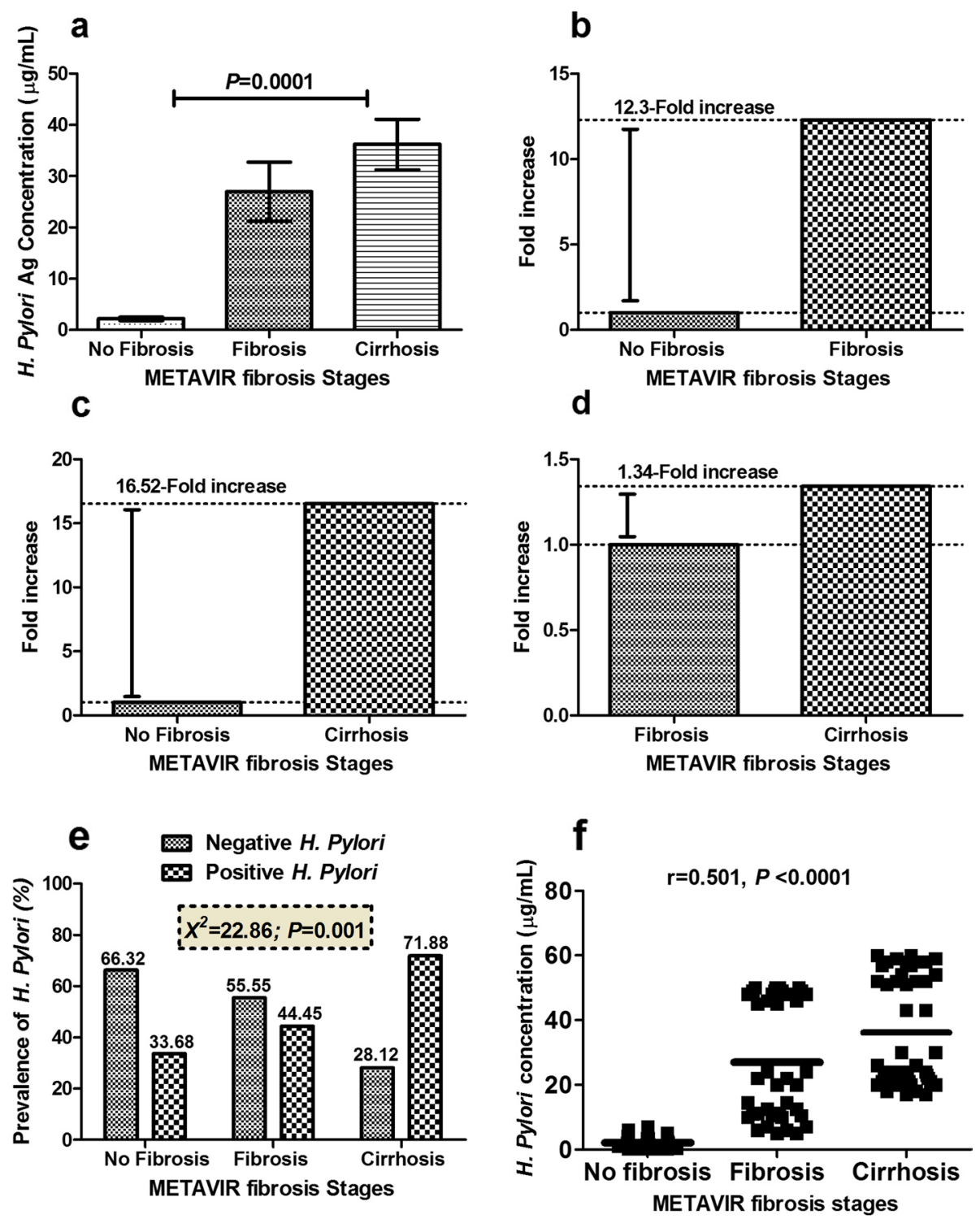

Fig. 2 Quantification of H. pylori antigen in addition to its observed fold changes in serum of chronic hepatitis C patients (a). The levels of 58-kDa H. pylori antigen among different METAVIR fibrosis stages $(\mathbf{b}, \mathbf{c}, \mathbf{d})$. The distribution of observed fold changes for 58-kDa H. pylori antigen among different METAVIR fibrosis stages (e). Prevalence of 58-kDa H. pylori antigen among patients with liver fibrosis and cirrhosis showing an increase in its detection rate with the progression of liver pathology (f). Scatter plot showing the distribution of 58-kDa H. pylori antigen with the progression of liver fibrosis. $P>0.05$ is considered non-significant, $P<0.05$ is considered significant, and $P<0.0001$ is considered extremely significant

that $H$. pylori infection may have a potential role in liverdisease progression.

\section{Discussion}

There is some evidence to suggest that $H$. pylori infection may be associated with an increased risk of gastric and extra-gastric malignancies [2,13-16]. Many of these studies were depending on detection of $H$. pylori antibodies in serum by ELISA [4, 17], histopathology methods [18], or on detection of bacterial deoxyribonucleic acid
(DNA) in tumor tissues using polymerase chain reaction technique (PCR) [5]. Clearly, the use of antibodiesbased methods can lead to ambiguous results especially since antibodies can be detectable a long time after the bacteria have ceased to colonize the gut [19]. The present work preferred to use $H$. pylori antigen-based method in order to examine the association between $H$. pylori infection and different liver diseases. In general, more efforts were performed for identifying various $H$. pylori antigens-based western blot technique, e.g., $125 \mathrm{kDa}$ 
Table 1 Comparison between laboratory characteristics of individuals included in this work based on Helicobacter pylori (H. pylori) infection $(n=249)$

\begin{tabular}{|c|c|c|c|}
\hline Variables $^{\mathrm{a}}$ & $\begin{array}{l}\text { Group I }(n=131) \\
\text { Non H. pylori infection }\end{array}$ & $\begin{array}{l}\text { Group II }(n=118) \\
\text { H. pylori infection }\end{array}$ & $P$ value \\
\hline Sex (male/female) & $97 / 34$ & $82 / 36$ & 0.481 \\
\hline $\operatorname{ALT}(\mathrm{U} / \mathrm{L})$ & $34.92 \pm 2.28$ & $48.78 \pm 3.00$ & $<0.0001^{*}$ \\
\hline AST (U/L) & $35.48 \pm 2.50$ & $51.97 \pm 3.35$ & $<0.0001^{*}$ \\
\hline Total bilirubin (mg/dl) & $1.00 \pm 0.15$ & $1.31 \pm 0.10$ & 0.087 \\
\hline Albumin $(\mathrm{g} / \mathrm{L})$ & $4.05 \pm 0.09$ & $3.31 \pm 0.07$ & $<0.000^{*}$ \\
\hline Platelets $\left(10^{9} / \mathrm{L}\right)$ & $235.87 \pm 11.42$ & $182.77 \pm 10.76$ & $0.001^{*}$ \\
\hline AFP (U/L) & $10.79 \pm 2.47$ & $35.44 \pm 10.07$ & $0.013^{*}$ \\
\hline
\end{tabular}

Variables were expressed as mean \pm SEM

a Reference values: alanine aminotransferase (ALT) (male up to $41 \mathrm{U} / \mathrm{L}$, female up to $31 \mathrm{U} / \mathrm{L}$ ); aspartate aminotransferase (AST) (male up to $37 \mathrm{U} / \mathrm{L}$, female up to $31 \mathrm{U} / \mathrm{L}$ ); total bilirubin up to $1 \mathrm{mg} / \mathrm{dL}$; albumin 3.8-5.4 g/dL; platelet count 150-400 × 109 $/ \mathrm{L}$; alpha fetoprotein (AFP) up to $10 \mathrm{U} / \mathrm{L}$

${ }^{*} P<0.05$ is considered significant

Table 2 Comparison between laboratory characteristics of individuals included in this work based on METAVIR fibrosis stages $(n=$ 249)

\begin{tabular}{|c|c|c|c|c|c|c|}
\hline \multirow[t]{2}{*}{ Variables $^{\mathrm{a}}$} & \multirow{2}{*}{$\begin{array}{l}\text { Group I }(n=95) \text {, } \\
\text { no fibrosis }\end{array}$} & \multirow[t]{2}{*}{ Group II $(n=90)$, fibrosis } & \multirow{2}{*}{$\begin{array}{l}\text { Group III ( } n= \\
64) \text {, cirrhosis }\end{array}$} & \multicolumn{3}{|c|}{ Tukey's post hoc test } \\
\hline & & & & Group I vs. II & Group I vs. III & Group II vs. III \\
\hline Age (year) & $32.26 \pm 0.65$ & $43.32 \pm 1.21$ & $54.00 \pm 2.08$ & $<0.0001^{*}$ & $<0.0001^{*}$ & $<0.0001^{*}$ \\
\hline $\mathrm{ALT}(\mathrm{U} / \mathrm{L})$ & $22.36 \pm 0.92$ & $54.07 \pm 2.45$ & $67.87 \pm 3.59$ & $<0.0001^{*}$ & $<0.0001^{*}$ & $<0.0001^{*}$ \\
\hline AST (U/L) & $24.09 \pm 1.23$ & $56.87 \pm 3.39$ & $68.97 \pm 4.35$ & $<0.0001^{*}$ & $<0.0001^{*}$ & $0.014^{*}$ \\
\hline Total bilirubin (mg/dl) & $0.71 \pm 0.03$ & $1.79 \pm 0.30$ & $1.78 \pm 0.18$ & $<0.0001^{*}$ & $<0.0001^{*}$ & 1.00 \\
\hline Albumin (g/L) & $3.88 \pm 0.07$ & $3.48 \pm 0.17$ & $2.97 \pm 0.11$ & $0.030^{*}$ & $<0.0001^{*}$ & $0.030^{*}$ \\
\hline Platelets $\left(10^{9} / \mathrm{L}\right)$ & $240.06 \pm 5.43$ & $172.03 \pm 22.24$ & $130.67 \pm 21.77$ & $0.015^{*}$ & $<0.0001^{*}$ & 0.259 \\
\hline $\operatorname{AFP}(U / L)$ & $1.35 \pm 0.06$ & $37.54 \pm 6.98$ & $94.18 \pm 23.95$ & $0.001^{*}$ & $<0.0001^{*}$ & $<0.0001^{*}$ \\
\hline
\end{tabular}

Variables were expressed as mean \pm SEM

${ }^{a}$ Reference values: alanine aminotransferase (ALT) (male up to $41 \mathrm{U} / \mathrm{L}$, female up to $31 \mathrm{U} / \mathrm{L}$ ); aspartate aminotransferase (AST) (male up to $37 \mathrm{U} / \mathrm{L}$, female up to $31 \mathrm{U} / \mathrm{L}$ ); total bilirubin up to $1 \mathrm{mg} / \mathrm{dL}$; albumin 3.8-5.4 g/dL; platelet count 150-400 $\times 10^{9} / \mathrm{L}$; alpha fetoprotein (AFP) up to $10 \mathrm{U} / \mathrm{L}$

${ }^{*} P<0.05$ is considered significant

Table 3 Levels of laboratory parameters in different METAVIR fibrosis stages in patients with hepatitis C virus mono-infection and HCV/Helicobacter pylori (H. pylori) co-infection

\begin{tabular}{|c|c|c|c|c|c|c|c|c|c|}
\hline \multirow[t]{2}{*}{ Variables $^{\mathrm{a}}$} & \multicolumn{3}{|c|}{ No fibrosis (F0) } & \multicolumn{3}{|c|}{ Liver fibrosis (F1-F3) } & \multicolumn{3}{|l|}{ Cirrhosis (F4) } \\
\hline & $\begin{array}{l}\text { Group } \mathrm{I}^{\mathrm{b}}(n \\
=63)\end{array}$ & $\begin{array}{l}\text { Group } I^{b}(n \\
=32)\end{array}$ & ${ }^{*} P$ value & $\begin{array}{l}\text { Group } \mathrm{I}^{\mathrm{b}}(n \\
=50)\end{array}$ & $\begin{array}{l}\text { Group } I^{b}(n \\
=40)\end{array}$ & ${ }^{*} P$ value & 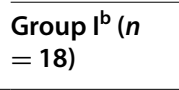 & $\begin{array}{l}\text { Group } I^{b}(n \\
=46)\end{array}$ & ${ }^{*} P$ value \\
\hline Age (year) & $32.05 \pm 0.91$ & $32.50 \pm 0.92$ & 0.732 & $40.58 \pm 1.55$ & $46.60 \pm 1.66$ & $0.011^{*}$ & $55.89 \pm 3.87$ & $53.23 \pm 2.50$ & 0.570 \\
\hline $\mathrm{ALT}(\mathrm{U} / \mathrm{L})$ & $19.50 \pm 0.90$ & $25.75 \pm 1.51$ & $<0.001^{*}$ & $49.28 \pm 2.36$ & $60.05 \pm 4.80$ & $0.027^{*}$ & $60.11 \pm 5.64$ & $70.91 \pm 4.39$ & $0.040^{*}$ \\
\hline AST (U/L) & $19.42 \pm 0.85$ & $29.63 \pm 2.11$ & $<0.001^{*}$ & $49.20 \pm 2.94$ & $66.45 \pm 6.14$ & $0.010^{*}$ & $65.17 \pm 6.66$ & $70.46 \pm 5.52$ & $0.046^{*}$ \\
\hline $\begin{array}{l}\text { T. bilirubin } \\
(\mathrm{mg} / \mathrm{dl})\end{array}$ & $0.58 \pm 0.03$ & $0.86 \pm 0.04$ & $<0.001^{*}$ & $1.73 \pm 0.23$ & $1.84 \pm 0.56$ & 0.868 & $1.72 \pm 0.44$ & $1.81 \pm 0.19$ & 0.817 \\
\hline Albumin (g/L) & $4.25 \pm 0.07$ & $3.45 \pm 0.08$ & $<0.001^{*}$ & $3.57 \pm 0.30$ & $3.41 \pm 0.21$ & 0.666 & $3.23 \pm 0.20$ & $2.89 \pm 0.12$ & 0.136 \\
\hline Platelets $\left(10^{9} / \mathrm{L}\right)$ & $239.5 \pm 7.2$ & $240.7 \pm 8.4$ & 0.920 & $200.2 \pm 35.1$ & $143.9 \pm 24.5$ & 0.224 & $245.5 \pm 121.5$ & $110.7 \pm 13.6$ & $0.025^{*}$ \\
\hline $\operatorname{AFP}(\mathrm{U} / \mathrm{L})$ & $1.04 \pm 0.08$ & $1.70 \pm 0.05$ & $<0.001^{*}$ & $26.38 \pm 3.93$ & $71.04 \pm 19.71$ & $0.003^{*}$ & $36.14 \pm 4.87$ & $106.96 \pm 22.39$ & $0.030^{*}$ \\
\hline
\end{tabular}

Variables were expressed as mean \pm SEM

${ }^{a}$ Reference values: alanine aminotransferase (ALT) (male up to $41 \mathrm{U} / \mathrm{L}$, female up to $31 \mathrm{U} / \mathrm{L}$ ); aspartate aminotransferase (AST) (male up to $37 \mathrm{U} / \mathrm{L}$, female up to $31 \mathrm{U} / \mathrm{L}$ ); total bilirubin (T. bilirubin) up to $1 \mathrm{mg} / \mathrm{dL}$; albumin 3.8-5.4 g/dL; platelet count $150-400 \times 10^{9} / \mathrm{L}$; alpha fetoprotein (AFP) up to $10 \mathrm{U} / \mathrm{L}$

${ }^{b}$ Group I: hepatitis C virus mono-infection; Group II: hepatitis C virus/Helicobacter pylori co-infection

${ }^{*} P<0.05$ is considered significant 
CagA antigen and $87 \mathrm{kDa}$ VacA antigen [20]. The 58-kDa H. pylori antigen was identified in serum of patients with different gastric and duodenal diseases. The molecular mass of the serum antigen is similar to the $58-\mathrm{kDa}$ fragment of the $87-\mathrm{kDa}$ cytotoxin domain of the VacA protein, the subunit cellular antigen of the native $H$. pylori catalase, and the $H$. pylori catalase gene product [9]. In this work, $H$. pylori antigen enabled the correct identification of $H$. pylori patients showing an AUC of 0.946 with $90 \%$ sensitivity and $90 \%$ specificity. These results are almost matching with those of Attallah et al. [9] and Kabir [21] who showed comparable sensitivity (91-98\%) and specificities (83-100\%). So, using a 58-kDa H. pylori antigen-based method was accurate enough for studying the $H$. pylori association with different gastrointestinal cancers. On the other hand, we also aimed to investigate the $58-\mathrm{kDa} H$. pylori antigen in sera of patients with different liver pathology to assess the potential cofactor role of $\mathrm{H}$. pylori infection in the progression of $\mathrm{HCV}$-related chronic liver disease. In this work, 58-kDa H. pylori antigen was positive in $71.88 \%$ of liver cirrhosis patients compared with $44.45 \%$ in liver fibrosis patients. Other studies have evidenced of high seroprevalence of $H$. pylori antibodies among cirrhotic patients [4, 13]. Our results are very close to that of Dore et al. [13] who detected antiH. pylori IgG in serum of $39 \%$ chronic hepatitis and $58 \%$ liver cirrhosis patients. In addition, Rocha et al. [5] found that Helicobacter DNA was high $68 \%$ in liver tissue of patients with liver cirrhosis. It was suggested that these bacteria could be implicated in the progression of liver disease to cirrhosis and hepatocellular carcinoma (HCC). In addition and consistent to our findings, Wang et al. found a strong association between $H$. pylori and chronic hepatitis $\mathrm{C}$, particularly during the $\mathrm{HCV}$ progression stage [22]. Surprisingly, Wang et al. showed that the odds ratio for cirrhosis was 4.4 which was almost similar to that obtained in our work. Huang et al. [23] postulated that it is possible for $H$. pylori to invade the liver and gall bladder via the bile duct. Many theories have been proposed to explain the mechanism of $H$. pylori involvement in the pathogenesis and progression of cirrhosis, particularly in HCV-infected individuals [24]. Enteric Helicobacter species have been found to produce toxins that might cause hepatocellular damage in vivo. Many studies have tried to explain the mechanisms by increased levels of proinflammatory cytokines such as interleukins 1,6 (IL-1, IL-6), tumor necrosis factor, and by the presence of lymphocytic mononuclear infiltrate and lymphoid follicle formation [23]. According to Abdel-Hady et al. [25], $H$. pylori infection might have a role in increasing the circulating levels of ammonia and endotoxins in cirrhotic patients, thus facilitating the onset of hepatic encephalopathy and of hepatic inflammation by stimulating the secretion of proinflammatory cytokines. As $H$. pylori infection increased hepatic AFP expression level and carbon tetrachloride $\left(\mathrm{CCL}_{4}\right)$ intoxication increased transforming growth factor-beta1 (TGF-b1) levels, both in serum and tissues, H. pylori chronic infection might contribute to HCC progression from CCL4-induced fibrosis or cirrhosis [26, 27].

\section{Conclusion}

In summary, this work showed that the elevated levels of $H$. pylori-antigen in $\mathrm{HCV} / \mathrm{H}$. pylori co-infection suggest increased susceptibility of co-infected patients for promoting hepatic fibrosis progression. This suggests the importance of $H$. pylori screening of patients with $\mathrm{CHC}$, particularly those with HCV-related cirrhosis, in order to choose the proper treatment.

Table 4 Prevalence of Helicobacter pylori (H. pylori) antigen in different METAVIR fibrosis stages in patients with hepatitis C

\begin{tabular}{|c|c|c|c|c|}
\hline \multirow[t]{2}{*}{ Fibrosis stages } & \multicolumn{2}{|c|}{ H. pylori prevalence } & \multirow[t]{2}{*}{ Odds ratio, $P$} & \multirow[t]{2}{*}{$X^{2}, P$} \\
\hline & Negative & Positive & & \\
\hline \multicolumn{5}{|c|}{ No fibrosis $(F 0)$ vs. fibrosis (F1-F3) } \\
\hline F0 $(n=95)$ & $63(66.32 \%)$ & $32(33.68 \%)$ & $1.5, P>0.05$ & $2.3, P>0.05$ \\
\hline $\mathrm{F} 1-\mathrm{F} 3(n=90)$ & $50(55.55 \%)$ & $40(44.45 \%)$ & & \\
\hline \multicolumn{5}{|c|}{ Fibrosis (F1-F3) vs. cirrhosis (F4) } \\
\hline $\mathrm{F} 1-\mathrm{F} 3(n=90)$ & $50(55.55 \%)$ & $40(44.45 \%)$ & $3.19, P=0.001^{*}$ & $11.4, P=0.001^{*}$ \\
\hline $\mathrm{F} 4(n=64)$ & $18(28.12 \%)$ & $46(71.88 \%)$ & & \\
\hline \multicolumn{5}{|c|}{ Non cirrhosis (FO-F3) vs. cirrhosis (F4) } \\
\hline F0-F3 $(n=185)$ & $113(61.08 \%)$ & $72(38.92 \%)$ & $4.0, P<0.0001^{*}$ & $20.71, P<0.0001^{*}$ \\
\hline $\mathrm{F} 4(n=64)$ & $18(28.12 \%)$ & $46(71.88 \%)$ & & \\
\hline
\end{tabular}

${ }^{*} P<0.05$ is considered significant 


\section{Abbreviations}

AFP: Alpha fetoprotein; ALP: Alkaline phosphatase; ALT: Alanine aminotransferase; AST: Aspartate aminotransferase; $\mathrm{CCL}_{4}$ : Carbon tetrachloride; $\mathrm{CHC}$ : Chronic hepatitis C; DNA: Deoxyribonucleic acid; H. pylori: Helicobacter pylori; HCC: Hepatocellular carcinoma; HCV: Hepatitis C virus; IL: Interleukins; PCR: Polymerase chain reaction technique; RNA: Ribonucleic acid; TGF-b1: Transforming growth factor-beta1.

\section{Acknowledgements}

The authors would like to thank staff of Biotechnology Research Center, New Damietta, Egypt, for kind help during the study. This study has been completely carried out at Biotechnology Research Center, New Damietta, Egypt.

\section{Authors' contributions}

AM and MF designed the study. AM and MS carried out literature search, designing, and editing of the manuscript. MS wrote and drew all the figures of the manuscript. SE, MM, and AA carried out the experimental work. All authors contributed to the final manuscript. All authors have read and approved the manuscript.

\section{Funding}

Not applicable.

Availability of data and materials

Not applicable.

\section{Declarations}

\section{Ethics approval and consent to participate}

The study protocol was approved by the Ethics Committee of the Mansoura University hospitals, Mansoura University, Egypt. The consent to participate was verbally and approved by Ethics Committee.

\section{Consent for publication}

The consent to publish had been taken verbally from each participant in this work.

\section{Competing interests}

The authors declare that they have no competing interests.

\section{Author details}

'Biotechnology Research Center, 23 July St., Industrial Zone, New Damietta 34517, Egypt. ${ }^{2}$ Faculty of Science, Zagazig University, Zagazig City, Egypt. ${ }^{3}$ Faculty of Science, Benha University, Benha City, Egypt.

Received: 27 June 2021 Accepted: 25 December 2021

Published online: 26 January 2022

\section{References}

1. Peek RM, Blaser MJ (2002) Helicobacter pylori and gastrointestinal tract adenocarcinomas. Nat Rev Cancer 2(1):28-37

2. Kim SS, Ruiz VE, Carroll JD et al (2011) Helicobacter pylori in the pathogenesis of gastric cancer and gastric lymphoma. Cancer Lett 305(2):228-238

3. Mamun AI M (2010) State of the globe: helicobacter pylori and hepatitis C together hamper health. J Global Infect Dis 2(1):1-3

4. Esmat G, El-Bendary M, Zakarya S et al (2012) Role of Helicobacter pylori in patients with HCV-related chronic hepatitis and cirrhosis with or without hepatocellular carcinoma: possible association with disease progression. J Viral Hepat 19(7):473-479

5. Rocha M, Avenaud P, Menard A et al (2005) Association of Helicobacter species with hepatitis $C$ cirrhosis with or without hepatocellular carcinoma. Gut. 54(3):396-401

6. Trikudanathan G, Philip A, Dasanu CA et al (2011) Association between Helicobacter pylori infection and pancreatic cancer. A cumulative metaanalysis. J Pancreas 12(1):26-31

7. Krüttgen A, Horz H-P, Weber-Heynemann J et al (2012) Study on the association of Helicobacter species with viral hepatitis-induced hepatocellular carcinoma. Gut Microbes 3(3):228-233
8. Eslick GD (2000) Helicobacter pylori infection transmitted sexually via oral-genital contact: a hypothetical model. Sex Transm Infect 76(6):489-492

9. Attallah AM, Ismail H, Ibrahim GG et al (2004) Use of a novel enzyme immunoassay based on detection of circulating antigen in serum for diagnosis of Helicobacter pylori infection. Clin Diagn Lab Immunol 11(4):775-779

10. Mao H, Li R (2014) Changes in serum pepsinogen in patients with liver cirrhosis and portal hypertensive gastropathy. Nan Fang Yi Ke Da Xue Xue Bao 34(1):141-143

11. Queiroz DMM, Rocha AMC, Rocha GA et al (2006) Association between Helicobacter pylori infection and cirrhosis in patients with chronic hepatitis C virus. Dig Dis Sci 51(2):370-373

12. Goodman ZD (2007) Grading and staging systems for inflammation and fibrosis in chronic liver diseases. J Hepatol 47(4):598-607

13. Dore MP, Realdi G, Mura D et al (2002) Helicobacter infection in patients with HCV-related chronic hepatitis, cirrhosis, and hepatocellular carcinoma. Dig Dis Sci 47(7):1638-1643

14. Buso AG, Rocha HLOG, Diogo DM et al (2009) Seroprevalence of Helicobacter pylori in patients with colon adenomas in a Brazilian university hospital. Arq Gastroenterol 46(2):97-9101

15. Stolzenberg-Solomon RZ, Blaser MJ, Limburg PJ et al (2001) Helicobacter pylori seropositivity as a risk factor for pancreatic cancer. J Natl Cancer Inst 93(12):937-941

16. de Martel C, Llosa AE, Friedman GD et al (2008) Helicobacter pylori infection and development of pancreatic cancer. Cancer Epidemiol Biomark Prev 17(5):1188-1194

17. Strofilas A, Lagoudianakis EE, Seretis C et al (2012) Association of Helicobacter pylori infection and colon cancer. J Clin Med Res 4(3):172-176

18. Soylu A, Ozkara S, Alis H et al (2008) Immunohistochemical testing for Helicobacter pylori existence in neoplasms of the colon. BMC Gastroenterol 8:35-40

19. Jones M, Helliwell P, Pritchard C et al (2007) Helicobacter pylori in colorectal neoplasms: is there an aetiological relationship? World J Surg Oncol 5:51-57

20. Abdel-Raouf M, Attallah A, Omran M et al (2014) The detection of a Helicobacter pylori antigen in some different types of gastrointestinal cancers patients. J Am Sci 10(7s):19-24

21. Kabir S (2001) Detection of Helicobacter pylori in faeces by culture, PCR and enzyme immunoassay. J Med Microbiol 50(12):1021-1029

22. Wang J, Li W-T, Zheng Y-X et al (2016) The association between Helicobacter pylori infection and chronic hepatitis C: a meta-analysis and trial sequential analysis. Gastroenterol Res Pract 2016:8780695

23. Huang Y, Tian XF, Fan XG et al (2009) The pathological effect of Helicobacter pylori infection on liver tissues in mice. Clin Microbiol Infect 15(9):843-849

24. Ceelen L, Decostere A, Verschraegen G et al (2005) Prevalence of Helicobacter pullorum among patients with gastrointestinal disease and clinically healthy persons. J Clin Microbiol 43(6):2984-2986

25. Abdel-Hady H, Zaki A, Badra G et al (2007) Helicobacter pylori infection in hepatic encephalopathy: relationship to plasma endotoxins and blood ammonia. Hepatol Res 37(12):1026-1033

26. Ki MR, Goo MJ, Park JK et al (2010) Helicobacter pylori accelerates hepatic fibrosis by sensitizing transforming growth factor-beta1-induced inflammatory signaling. Lab Investig 90(10):1507-1516

27. Berasain C, Perugorria MJ, Latasa MU et al (2009) The epidermal growth factor receptor: a link between inflammation and liver cancer. Exp Biol Med 234(7):713-725

\section{Publisher's Note}

Springer Nature remains neutral with regard to jurisdictional claims in published maps and institutional affiliations. 